\section{Langzeittherapie mit COX-2-Hemmern}

Die Therapie mit klassischen nicht steroidalen Antirheumatika, die auch die Cyclooxygenase 1 ( $C O X_{1}$ ) hemmen, kann durch das Auftreten pseudoallergischer Reaktionen kompliziert werden. Die Einführung der COX-2-Hemmer brachte daher eine deutliche Risikominderung. Unbekannt war jedoch, ob das günstige Nebenwirkungsprofil auch bei einer Langzeittherapie erhalten bleibt.

\begin{abstract}
A llergologen der Universität Ankara, Türkei, unterzogen von 1997 bis 2003235 Patienten mit eindeutigen pseudoallergischen Reaktionen auf herkömmliche nicht steroidale Antirheumatika einer oralen Provokation mit einem oder mehreren COX-2-Hemmern. Es waren dies Nimesulid, Meloxicam, Celecoxib und Rofecoxib. Letztere Substanz wurde nach dem Bekanntwerden kardialer Nebenwirkungen nicht mehr eingesetzt.

Alle Provokationstestungen verliefen negativ. Die als verträglich ausge-
\end{abstract}

wiesenen COX-2-Hemmer wurden daraufhin zur weiteren Therapie verordnet. Regelmäßige Telefoninterviews durch erfahrene Allergologen konnten bei 87 der Patienten durchgeführt werden. Davon hatten sich bei 26 die Beschwerden so sehr gebessert, dass sie in der Lage waren, die Medikation ganz einzustellen. Die verbliebenen 61 wurden über einen Zeitraum von durchschnittlich mehr als 2 Jahren beobachtet. Sieben Patienten berichteten über Nebenwirkungen. Bei drei dieser Patienten verlief die orale Reexposition negativ.
Die übrigen vier standen hierfür nicht zur Verfügung und blieben ätiologisch ungeklärt.

Die COX-2-Inhibitoren bedeuten somit offenbar bezüglich pseudoallergischer Nebenwirkungen gegenüber den herkömmlichen nicht steroidalen Antirheumatika einen deutlichen Fortschritt. Dies gilt ganz besonders für Patienten, welche auf COX-1-Hemmer pseudoallergisch reagieren, aber weiter einer Schmerztherapie bedürfen. Jedoch sollte bei solchen Patienten der Verordnung eines COX-2-Hemmers sicherheitshalber eine orale Provokation vorausgehen. Tendenziell sollten aus allergologischer Sicht Analgetika mit hoher COX-2-Spezifität bevorzugt werden.

Dr. Dieter Bruchhausen, Wuppertal

\section{Literatur}

Celik G, Erkekol FO, Bavbek S, Dursun B, Misirligil Z. Long-term use and tolerability of cyclooxygenase-2 inhibitors in patients with analgesic intolerance. Ann Allergy Asthma Immunol 2005; 95: 33-7

\section{Kontaktallergien gegen Haarpflegemittel}

\author{
Auch in Großbritannien ist die Berufsgruppe der Friseure besonders \\ häufig von Kontaktallergien betroffen. Eine große Multizenterstudie \\ sollte herausfinden, welche Allergene aktuell für Friseure und deren \\ Kunden besonders relevant sind.
}

$\mathrm{N}$ eun britische Kontaktallergiezentren legten für die Dauer von 2-6 Jahren die Ergebnisse ihrer Hauttestungen in einer zentralen Datenbank ab. Ziel war es, herauszufinden, welche Allergene in welcher Häufigkeit in den verschiedenen Zentren getestet werden und welche Testkonzentrationen optimal sind, um falsch positive und falsch negative Testergebnisse möglichst zu vermeiden. Altbekannte aber auch neuere Haarpflegechemikalien wurden in die Testreihen einbezogen.

Die meisten Sensibilisierungen waren mit Abstand bei Haarfärbe- und Haarbleichmitteln zu verzeichnen. Das galt sowohl für die Friseure selbst als auch für deren Kunden. Die Institute einigten sich nach Abschluss der Untersuchung auf einen 10 Kontaktallergene umfassenden Testblock, der nunmehr von der British Contact Dermatitis Society empfohlen wird:

-2,5-Toluylendiamin,

- Ammoniumpersulfat,

- Para-Phenylendiamin,

- Glycerylmonothioglycolat,

-4-Aminophenol,

-3-Aminophenol,

- Hydrochinon,

-Captan,

- Ammoniumthioglycolat.

Das Para-Phenylendiamin wird wegen seiner hochgradig sensibilisierenden Eigenschaften heute auch in Großbritannien nicht mehr überall routinemäßig in Testreihen eingesetzt.

Dr. Dieter Bruchhausen, Wuppertal

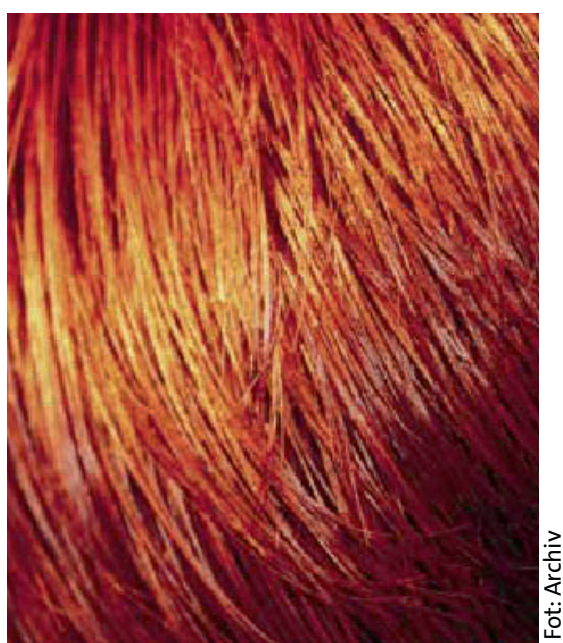

Gefärbte Haare liegen weiter im Trend, damit ist auch den Färbemitteln ein Spitzenplatz in der Hitliste der häufigsten Kontaktallergene bei Friseuren sicher.

\section{Literatur}

Katugampola RP, Statham BN, English JS, Wilkinson MM, Foulds IS, Green CM, Ormerod AD, Stone NM, Horne HL, Chowdhury MM. A multicentre review of the hairdressing allergens tested in the UK. Contact Dermatitis 2005; 53: 130-2 